\title{
MODULATION EFFECT OF MAGNETIC COROTATING TRAP ON 27-DAY COSMIC RAY VARIATION IN NOVEMBER-DECEMBER 2014
}

\author{
V.E. Sdobnov \\ Institute of Solar-Terrestrial Physics SB RAS, \\ Irkutsk, Russia,sdobnov@iszf.irk.ru
}

\section{M.V. Kravtsova}

Institute of Solar-Terrestrial Physics SB RAS, Irkutsk, Russia,rina@iszf.irk.ru

\author{
S.V. Olemskoy \\ Institute of Solar-Terrestrial Physics SB RAS, \\ Irkutsk,Russia,osv@iszf.irk.ru
}

\begin{abstract}
We study the 27-day cosmic-ray (CR) intensity variation occurring in November-December 2014, using ground-based measurements from the worldwide network of neutron monitors and GOES-15 satellites.

A determining factor in the considerable difference between amplitudes of the 27-day CR variation in November-December 2014 is shown to be significant changes in energy losses taking place when particles move in regular heliospheric electromagnetic fields. In
\end{abstract}

\section{INTRODUCTION}

An important feature of interplanetary space is its sector structure. This means that in the plane of the ecliptic there may be an even number of sectors with different directions of the radial component of the interplanetary magnetic field (IMF).

The IMF sector structure is associated with the existence of a neutral current layer separating the hemispheres in which the radial component has the opposite direction (since the magnetic flux through any closed surface is zero, the magnetic field in different regions of space should be oppositely directed). Every 22 year, the solar magnetic field changes sign (reversal). The current layer is located approximately in the solar equatorial plane and has a corrugated structure - it is deflected alternately to the north and south of the equator. As a result of the solar rotation, ripples of the current sheet are twisted into a spiral.

Earth, moving in interplanetary space, falls into sectors with different directions of the radial IMF component. Due to longitude and latitude gradients of the solar wind (SW) velocity, observed near the Sun, with distance from it there appear radial velocity gradients, which lead to the formation of collisionless shock waves near sector boundaries. Such waves occur at distances from 1 AU and can be observed to distances of several AU.

If an activity complex exists for several solar rotations, then due to the fact that the magnetic inhomogeneity seems to be anchored to a particular field line going out of this meridian, this inhomogeneity will rotate with the Sun. CR propagation in the heliosphere in the presence of such inhomogeneities causes a 27-day variation. The influence of corotating SW structures on CR behavior has been extensively studied [Lee et al., 2010; Modzelewska, Alania, 2013; Gil, Alania, 2016].

The amplitude of the 27-day variation of the CR neutron component, measured at sea level, is usually this period, there was a long-living corotating trap produced by a vast coronal hole in the south of the Sun in interplanetary space. Configuration of this trap induced the energy loss of $\sim 3-20 \mathrm{GeV}$ CRs, due to which ground-based neutron monitors recorded an abnormally large amplitude of the 27-day variation.

Keywords: cosmic-ray modulation, solar activity, 27-day variations.

within $\sim 0.7-1.0 \%$ of the quiet level [Dorman, 1963].

From the second half of 2014 until March 2015, CR stations of the worldwide network observed the 27-day variation of abnormally high amplitude [Gil, Mursula, 2015]. So, at the CR station Irkutsk (threshold rigidity $R=3.66 \mathrm{GW}$ ), located at an altitude of $433 \mathrm{~m}$, the peakto-peak amplitude of the 27-day CR variation in November-December 2014 was $\sim 8 \%$.

According to [http://www.solarmonitor.org], in the second half of 2014 in the south pole of the Sun there was a huge coronal hole, which led to an asymmetric magnetic configuration at middle and high heliolatitudes. The tilt angle of the current sheet in NovemberDecember was $\sim 52^{\circ}$ [http://wso.stanford.edu]. In addition, in that period the Sun reversed its polarity [http://wso.stanford.edu].

This paper interprets the abnormally high amplitude of the 27-day CR variation in NovemberDecember 2014.

\section{DATA AND METHOD}

The analysis is based on hourly averaged ground measurements from 39 neutron monitor stations of the worldwide network. We have also used data from the GOES-15 spacecraft [http://satdat.ngdc.noaa.gov./sem/goes/data/new_avg].

The spectrographic global survey method [Dvornikov, Sdobnov, 2002; Dvornikov et al., 1983] was employed to obtain information about variations in angular and energy distribution of primary CRs outside Earth's magnetosphere, as well as about changes in the planetary system of geomagnetic cutoff rigidity for each observation hour. Modulation amplitudes were measured from the background level of November 28, 2014. Using information obtained from data from the worldwide network of CR stations and from GOES-15 measurements of 
protons in Earth's orbit, [Dvornikov, Sdobnov, 2002], we calculated CR proton spectra for certain moments of the events under study. To calculate the CR spectra, we employed the expression derived using the model of CR modulation by regular electromagnetic fields of the heliosphere [Dvornikov et al., 2013].

\section{RESULTS AND CONCLUSIONS}

The huge coronal hole in the second half of 2014 and early 2015 [http://www.solarmonitor.org] gave rise to quasi-stationary high-speed solar plasma streams in interplanetary space. During propagation, the high-speed stream catches up with a slow stream. Their interaction produces a magnetic trap, which rotates with the Sun at 27-day intervals, causing 27-day variations in $\mathrm{CR}$ intensity.

According to [Krymsky, 1969], these CR variations require the presence of ordered IMF; therefore a largely important factor in CR modulation with 27 day periodicity may be a change of their energy when they move in regular electromagnetic fields of the heliosphere. The energy change is, in turn, determined by the intensity and nature of electric fields in the interplanetary medium and the time of interaction of particles with these fields [Dvornikov et al., 2013], which mainly depends on IMF magnetic traps. In this approach, the energy change is described by the expression

$$
\Delta \varepsilon=\frac{z e \Omega B_{0} r_{0}^{2}}{c}\left(1-\cos \lambda_{E}\right)=z e U,
$$

where $U=\Omega B_{0} r_{0}^{2}\left(1-\cos \lambda_{E}\right)$ is the electric field potential at a heliolatitude $\lambda_{E}, \Omega$ is the angular velocity of solar rotation, $B_{0}$ is the average magnetic field strength at $r_{0}$. The CR rigidity spectrum in Earth's orbit is described by the expression [Dvornikov et al., 2013]

$$
J(r)=A \frac{\left(\varepsilon^{2}-\varepsilon_{0}^{2}\right)^{3 / 2}}{\varepsilon\left[(\varepsilon+\Delta \varepsilon)^{2}-\varepsilon_{0}^{2}\right]}\left(\frac{\varepsilon+\Delta \varepsilon}{T_{0}+\varepsilon_{0}}\right)^{-\gamma},
$$

where $\varepsilon$ is the total energy of particles with rigidity $R ; \Delta \varepsilon$ is $\varepsilon$ changes in electromagnetic fields of the heliosphere; $\varepsilon_{0}$ is the rest energy; $T_{0}$ is the kinetic energy of particles at which the $\mathrm{CR}$ intensity of the corresponding galactic rigidity is $A ; \gamma$ is the spectral index of the galactic spectrum.

We can see that the particle energy losses at a potential electric field do not depend on particle rigidity and with the characteristic IMF strength $(\sim 5 \mathrm{nT})$ are $\sim 0.2 \mathrm{GeV}$.

When IMF has a structure different from the spiral one (e.g., in the presence of loop structures or magnetic clouds, i.e. magnetic traps), $\Delta \varepsilon$ values depend on the strength of magnetic fields of these structures and SW velocity and exceed $0.2 \mathrm{GeV}$ by almost an order of magnitude [Dvornikov et al., 2013].

Figure 1 shows SW and IMF parameters. The periods are well defined when IMF is antisunward and when it reverses the direction, i.e. the IMF sector structure is observed.

According to spacecraft measurements of SW parameters [https://omniweb.gsfc.nasa.gov/ow.html], the average
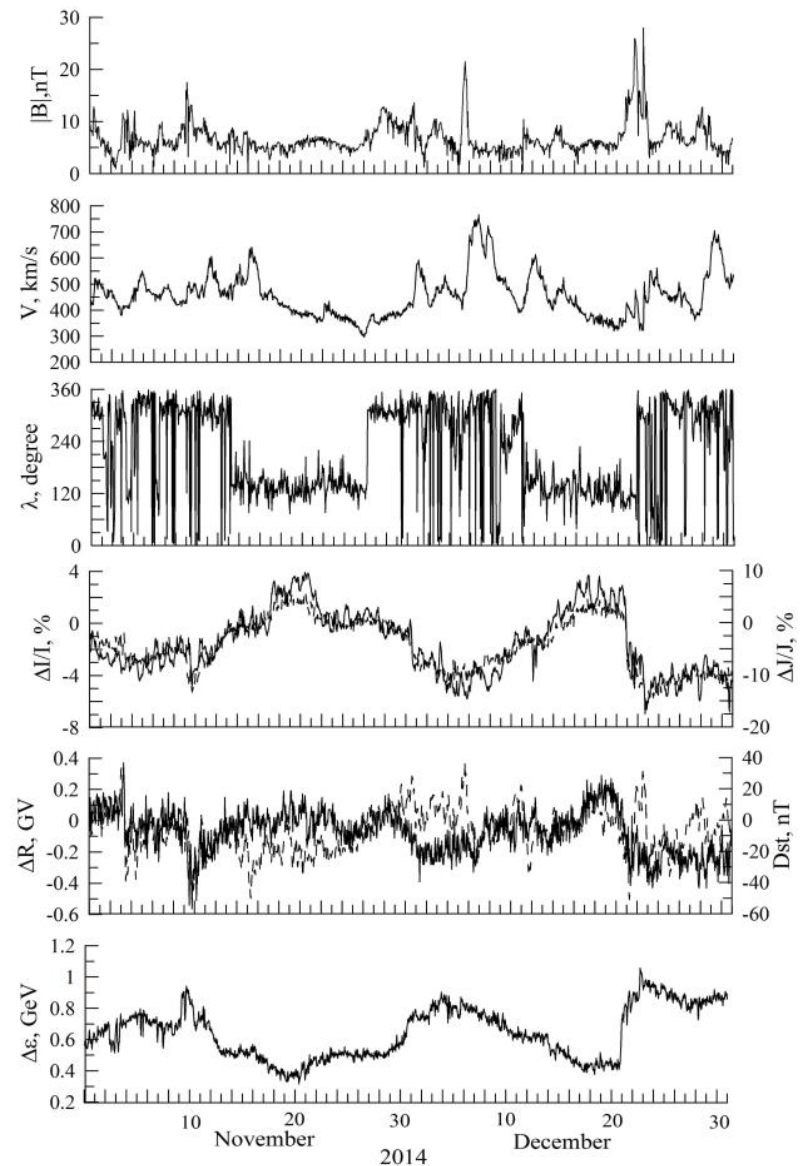

Figure 1. $|B|$ is the modulus of IMF strength, $\lambda$ is the longitudinal angle of IMF orientation, $V$ is the SW velocity, $\Delta I / I$ is the amplitude of $C R$ variations at Irkutsk station $\left(R_{\mathrm{c}}=3.66 \mathrm{GV}\right)$ (solid line), $\Delta J / J$ is the amplitude of $\mathrm{CR}$ variations with $10 \mathrm{GV}$ rigidity in Earth's orbit (dashed line), $\Delta R$ is a change in the CR geomagnetic cutoff rigidity in Irkutsk (solid line), Dst is the geomagnetic activity index (dashed line); $\Delta \varepsilon$ is a change of particle energy in electromagnetic fields of the heliosphere

IMF modulus in the sector with the sunward magnetic field in 2014 was larger $(\sim 7.2 \mathrm{nT})$ than that in the sector with the antisunward magnetic field ( 6.2 nT).

Figure 1 shows that the amplitude of the 27-day variation in CR neutron component in Irkutsk in November-December 2014 is $\sim \pm 4 \%$, which is much larger than the commonly observed amplitude.

Such a large difference between amplitudes of the variations is due to a different amount of energy losses by CR particles in electromagnetic fields of the heliosphere during this period. These losses ranged from $\sim 0.3$ to $\sim 1.1 \mathrm{GeV}$. This value in the 27-day cycle is generally from $\sim 0.2$ to $\sim 0.5-0.7 \mathrm{GeV}$. We can conclude that a determining factor in the observed large-amplitude 27-day CR variations in November-December 2014 is significant variations in energy losses when particles are moving in regular electromagnetic fields of the heliosphere, and the fact that these losses in the sector with antisunward IMF are much less than those in the opposite sector.

To figure out in which energy range the maximum modulation occurs in different IMF sectors, we have calculated average differential CR spectra in sectors with sunward $\left(J_{-}\right)$and antisunward $\left(J_{+}\right)$IMF for November- 


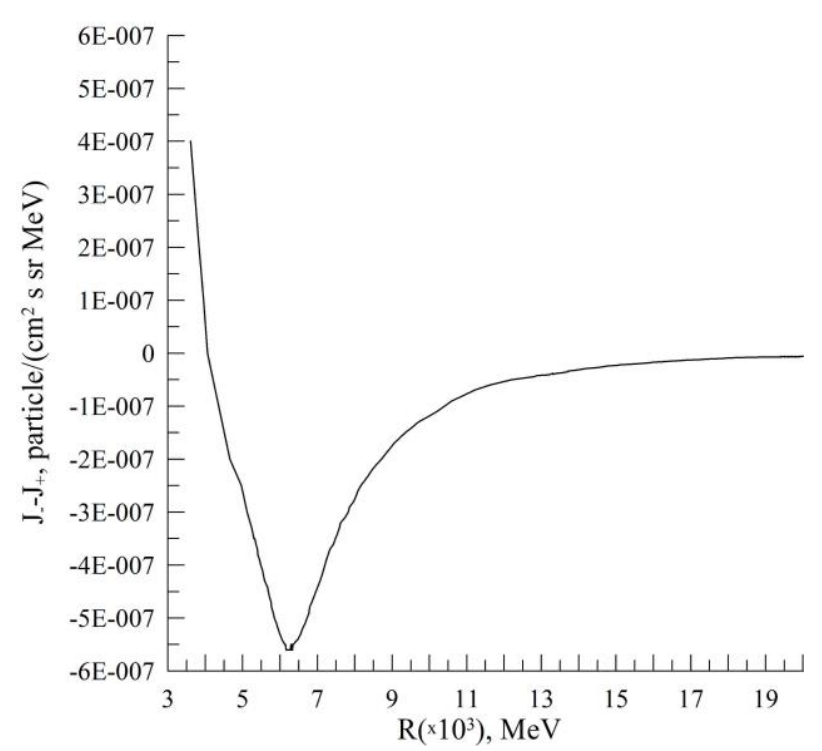

Figure 2. $J_{-}-J_{+}$is the difference between average differential CR spectra in sectors with sunward $\left(J_{-}\right)$and antisunward $\left(J_{+}\right)$IMF

December 2014. Figure 2 shows the difference between these spectra $J_{-}-J_{+}$. It is apparent that in the energy range from $\sim 3$ to $20 \mathrm{GeV}$, where neutron monitors have the highest sensitivity, modulation prevails in the sectors with sunward IMF.

Presumably during this period in interplanetary space under the influence of the huge coronal hole in the south of the Sun there emerged a long-lived corotating trap of such a configuration at which $\sim 3-20 \mathrm{GeV}$ CRs lost their energy most effectively. As a result, groundbased measurements of CR intensity by neutron monitors show an abnormally large amplitude of 27-day variations.

The work was carried out under the state task for 2018 No. 007-00163-18-00 from January 12, 2018 with the equipment of Center for Common Use "Angara" and with the Unique Research Facility "Russian national ground network of cosmic ray stations".

\section{REFERENCES}

Dorman L.I. Variatsii kosmicheskikh luchei I issledovanie kosmosa [Cosmic ray: variations and space exploration] Moscow, AN SSSR Publ., 1963. 1028 p.

Dvornikov V.M., Sdobnov V.E. Variations in the rigidity spectrum and anisotropy of cosmic rays at the period of Forbush effect on 12-15 July. Intern. J. Geomagn. Aeron. 2002, vol. 3, no. 3, p. 217.

Dvornikov V.M., Sdobnov V.E., Sergeev A.V. Analysis of cosmic ray pitch-angle anisotropy during the June 1972 Forbush effect by method of spectrografic global survey. Proc. $18^{\text {th }}$ ICRC. Bangalore. India. 1983, vol. 3, p. 249.

Dvornikov V.M., Kravtsova M.V., Sdobnov V.E. Diagnostics of electromagnetic characteristics of the interplanetary medium based on cosmic ray effects. Geomagnetism and Aeronomy. 2013, vol. 53, iss. 4, pp. 430-440.

Gil A., Alania M.V. Energy spectrum of the recurrent variation of galactic cosmic rays during the solar minimum of cycles 23/24. Solar Phys. 2016, vol. 291, iss. 6, pp. 1877-1886. DOI: 10.1007/ s11207-016-0924-z.

Gil A., Mursula K. Exceptionally strong variation of galactic cosmic ray intensity at solar rotation period after tr- maximum of solar cycle 24. Proc. the $34^{\text {th }}$ International Cosmic Ray Conference, 30 July - 6 August, 2015, The Hague, The Netherlands. PoS(ICRC2015)149.

Krymsky G.F. Modulyatsiya kosmicheskijh luchei v mezhplanetnom prostranstve [Cosmic ray modulation in the interplanetary medium]. Moscow, Nauka, 1969, $152 \mathrm{p}$.

Lee C.O., Luhmann J.G., de Pater I., Mason G.M., Haggerty D., Richardson I.G., Cane H.V., Jian L.K., Russell C.T., Desai M.I. Organization of energetic particles by the solarwind structure during the declining to minimum phase of solar cycle 23. Solar Phys. 2010, vol. 263, iss. 1-2, pp. 239261. DOI: $10.1007 / \mathrm{s} 11207-010-9556-\mathrm{x}$.

Modzelewska R., Alania M.V. The 27-day cosmic ray intensity variations during solar minimum 23/24. Solar Phys. 2013, vol. 286, iss. 2, pp. 593-607. DOI: 10.1007/s11207013-0261-4. 2018).

URL: http://www.solarmonitor.org (accessed September 8,

URL: http://wso.stanford.edu (accessed September 8, 2018).

URL: http://satdat.ngdc.noaa.gov./sem/goes/data/new_avg (accessed September 8, 2018)

URL: https://omniweb.gsfc.nasa.gov/ow.html (accessed September 8, 2018).

How to cite this article

Sdobnov V.E., Kravtsova M.V., Olemskoy S.V. Modulation effect of magnetic corotating trap on 27-day cosmic ray variation in NovemberDecember 2014. Solar-Terrestrial Physics. 2019. Vol. 5. Iss. 1. P. 11-13. DOI: 10.12737/stp-51201902. 